\title{
Entscheidungskriterium Plausibilität
}

Unser tägliches Handeln ist bestimmt von unserer eigenen Erfahrung und, wo diese nicht in befriedigendem Umfang zur Verfügung steht, vom Ergebnis interner Plausibilitätskontrollen. Dass Erfahrung eine notwendige, aber keine hinreichende Voraussetzung für richtige Entscheidungen ist, ist vielfach belegt. Erfahrung heisst, auf vergangene Ereignisse zurückgreifen zu können. Je grösser die Anzahl der einzelnen Erfahrungen ist, die dabei einfliesst, umso weniger ist davon auszugehen, dass die Erfahrung als Mittelwert dieser Einzelerfahrungen (statistisch gesehen) ein Ausreisser ist. Daraus lässt sich aber weder Zuverlässiges über die Eigenschaften einzelner künftiger Ereignisse noch über die eines Mittelwerts künftiger Ereignisse ableiten. Voraussetzung wäre nämlich, dass sich alle vergangenen wie zukünftigen Phänomene mathematisch im Sinne einer stetigen Funktion darstellen liessen. Anders ausgedrückt, alle müssten einem immanenten, invariablen Prinzip unterworfen sein, das weder durch (grundsätzlich) veränderliche äussere Einflüsse noch durch eine nichtstetige innere Evolution beeinflussbar ist.

Das aber gibt es in der Natur so nicht, im Gegenteil, es wird immer deutlicher, wie sehr die Natur durchdrungen ist vom Prinzip der nichtlinearen Dynamik, will heissen, kleine, zufällige Phänomene und Ereignisse können für den künftigen Lauf der Dinge in nichtvorhersagbarer Weise beeinflussend werden. Ein Beispiel hierfür haben wir täglich vor Augen - die Entwicklung an den Börsen und die (retrospektiven) Erklärungen der Fachleute. So plausibel sie klingen, so wenig verlässlich lässt sich der weitere Verlauf der Ereignisse daraus ableiten.

Was mich zum Aspekt der Plausibilität bringt. Diese ist im Beispiel der Analyse von Börsenkursen kein Garant dafür, dass der vom Analysten hergestellte Zusammenhang tatsächlich existiert bzw. dass er wirklich kausaler Natur ist. Im Bereich der Klassischen Naturheilkunde argumentieren wir nicht selten mit der vermeintlichen Trumpfkarte der Plausibilität. Sicher, die Erklärungsmodelle für wichtige Verfahren sind zwanglos nachvollziehbar, aber sind sie deshalb auch automatisch richtig? Und, müssen Verfahren, für die weniger plausible Modelle angeboten werden, deshalb notwendigerweise automatisch weniger richtig, müssen sie deshalb fragwürdiger sein?

Macht man sich auf die Suche, findet man in der Medizin in grosser Zahl Beispiele, dass das einem Verfahren zu Grunde gelegte Modell im Laufe der Zeit Wandlungen unterworfen war, ohne dass das Verfahren selbst Schaden genommen hätte. Andere Naturwissenschaftler scheinen sich da leichter zu tun. So werden einige Eigenschaften des Lichts plausibel, wenn als Modell ein Teilchenstrom, und andere, wenn Wellencharakter unterstellt wird, und wir können das Licht nutzen, ohne dass wir seine wahre Natur kennen. Heute wissen wir, dass Phänomene, für die uns die Natur nicht mit einem geeigneten Sinnesorgan ausgestattet hat, ohne entsprechende technische Hilfsmittel nicht korrekt erklärbar sind (wie etwa Radioaktivität, Infrarotlicht oder Ultraschall).

Deshalb plädiere ich dafür, im professionellen Umfeld andere, eben professionellere Massstäbe anzulegen als privat. Deshalb erlaube ich mir den Luxus, Plausibilität zur Grundlage von Entscheidungen einzusetzen, nur privat. Deshalb gilt ansonsten: Fakten (bzw. deren Fehlen) sind Grundlage und Massstab für Entscheidungen.

K.-L. Resch, Bad Elster

\section{KARGER}

Fax +497614520714 (c) 2000 S. Karger GmbH, Freiburg

E-mail Information@Karger Accessible online at:

www.karger.com 